\section{Abnormal Placentation: Challenges and Keys for Success}

\author{
Ali S Faris ${ }^{1 *}$, Qutaiba A Tawfic ${ }^{2}$ and Leo Jeyaraj ${ }^{3}$ \\ ${ }^{1}$ Department of Anesthesiology and Pain Management, Tawam Hospital, \\ Al Ain, UAE \\ ${ }^{2}$ Department of Anesthesia and Perioperative Medicine, Western \\ University, London Ontario, Canada \\ ${ }^{3}$ Department of Anesthesiologist, The Ottawa Hospital, Ottawa, ON, \\ Canada
}

\begin{abstract}
The incidence of various degrees of abnormally adherent placenta is increasing, making it one of the common causes of obstetrical hemorrhage and it might account for up to half of all cesarean hysterectomies and contributing to maternal and perinatal morbidity. We report on the successful management of a complex case of placenta percreta at our hospital, where the placenta invaded the urinary bladder and the small bowel. We further share our management strategies and extrapolate the keys to success in the management of similar cases of abnormal placentation.

Keywords: Abnormal placentation; Cesarean; Hemorrhage
\end{abstract}

\section{Introduction}

Abnormal placentation is an increasing life threatening condition associated with many complications that increase the maternal and perinatal morbidity and mortality. The rate of abnormal placentation is increasing in concurrence with rising cesarean deliveries and associated with the presence of other risk factors like previous uterine scars, previa, and advanced maternal age $[1,2]$. It is one of the most common causes of obstetrical hemorrhage and the indication for cesarean hysterectomies in about $50 \%$ of the emergency peripartum hysterectomies $[3,4]$.

\section{Case Description}

A 37 year old Gravida3/Para 2 with 2 previous cesarean deliveries was admitted with hematuria at 29 weeks gestational age. Ultrasound examination revealed complete placenta previa with suspicion of percreta. The MRI showed evidence of placental invasion into the urinary bladder and small bowel. The case was analyzed by a multi-disciplinary team including high risk obstetricians,

*Corresponding author: Ali S Faris, Department of Anesthesiology and Pain Management, Tawam Hospital, Al Ain, UAE, Tel: 97137649964 ; E-mail: alfaris@hotmail.ca

Citation: Faris AS, Tawfic QA, Jeyaraj L (2014) Abnormal Placentation: Challenges and Keys for Success. J Anesth Clin Care 1: 003.

Received: September 13, 2014; Accepted: November 20, 2014; Published: December 09, 2014 urologists and general surgeons. The plan involved elective cesarean section and hysterectomy at 32 weeks gestational age. As a standard protocol for management of such cases, the internal iliac arteries were planned to be catheterized pre-operatively by Interventional Radiology and balloon catheters were to be placed for safety. The patient declined this procedure later due to concerns over fetal radiation exposure and due to her needle phobia. The anesthetic plan consisted of combined epidural - general anesthetic with invasive monitoring.

The epidural catheter was placed while the patient awake in the sitting position, at L3-4 level following which, general anesthesia was induced uneventfully using rapid sequence induction with thiopental and succinylcholine and the airway was secured with size 7 cuffed endotracheal tube. Anesthesia was maintained with sevoflurane in $\mathrm{O}_{2} / \mathrm{N}_{2} \mathrm{O}$ (50:50) mixture. The invasive lines (Arterial and Central Venous Catheter) were placed without difficulty after delivery of a healthy baby. The internal iliac arteries were subsequently ligated and a sub-total hysterectomy performed with some placental tissue left in-situ on the bladder and left pelvic wall. The estimated blood loss was 11 litres; 21 units of PRBCs were transfused intra-operatively along with 7 units FFP, 2 pooled platelets, 1 pooled cryoprecipitate in addition to 2 doses of tranexamic acid (of 1 gram each) and 2 doses of recombinant Factor VIIa ( $2.4 \mathrm{mg}$ each). At the end of surgery (total operating time of 5 hours), the patient body temperature was $36^{\circ} \mathrm{C}, \mathrm{Hb} 68 \mathrm{~g} / \mathrm{L}, \mathrm{pH} 7.27, \mathrm{BE}-9$ and INR 0.8. The patient was kept sedated, intubated and transferred to the ICU where she was kept on ventilator overnight and she was successfully extubated on the next day (postoperative day 1) after being in stable hemodynamic, metabolic and respiratory condition. She was then discharged from the ICU on the second postoperative day to a regular postpartum bed where she stayed for 6 days in stable condition and without significant complications and was discharged home. The baby's Apgar scores at 1 and 5 minutes were $7 \& 10$ respectively. He was admitted to the NICU for monitoring and was discharged later in a good condition.

\section{Discussion}

With the increasing incidence of both pregnancies and cesarean delivery, this life threatening condition is becoming more common. Abnormal placental attachment has an incidence of 3 per 1000 deliveries and it accounts for up to $50 \%$ of all emergency peripartum hysterectomies with a maternal mortality rate approximating $7 \%$. The reason behind this condition is that the trophoblast breaks through the decidua and invades the uterine wall to various degrees $[1,5]$. The cases of abnormal placental attachment are divided into placenta accreta $(75 \%)$, increta $(18 \%)$, and percreta $(7 \%)$. Common risk factors for abnormal placentation include placenta previa with or without previous uterine surgery, previous Cesarean Section (CS), previous myomectomy, prior uterine curettage or endometrial ablation and increasing maternal age. Placenta percreta is associated with many complications that increase the maternal and perinatal morbidity and mortality and these complications may include damage to bladder, bowel and other pelvic structures, massive hemorrhage and its consequences like coagulopathy, ARDS and multiorgan failure $[5,6]$. 
Regional anesthesia (epidural and spinal anesthesia) can still be used in cases of abnormal placentation; however, some studies have shown some preference to general anesthesia to the risk of significant bleeding, hypotension and coagulopathy and the need to protect the airway. It is crucial that the skilled anesthesiologist take all the preparatory steps to manage the anticipated massive bleeding, including efficient intravenous access, prepared blood products and blood bank cooperation with the implication of blood conservation techniques, invasive blood pressure and central venous pressure monitoring, and the arrangement of postoperative intensive care unit admission $[7,8]$. Recently, a possible beneficial role of recombinant factor VIIa was described in the management of severe postpartum hemorrhage $[9,10]$. Tranexamic acid is one of the treatment choices that could be of benefit in preventing or reducing massive postpartum bleeding, although the evidence is not yet strong $[11,12]$.

In a review of the published cases, Clausen et al., compared the complications of three commonly used surgical approaches for the management of severe placenta percreta: local resection, hysterectomy or leaving the placenta in situ. They concluded that conservative strategy (leaving the placenta in situ) appears to be linked with severe long-term complications of hemorrhage and infection while Local resection was found to have fewer complications within 24 hours postoperatively compared with the other two surgical approaches [13].

The keys for successful management of parturients with abnormal placentation may include: assessment of risk factors and prenatal diagnosis, multidisciplinary care team approach, location of surgery and logistics, early involvement of expert anesthesiologist to be part of proactive team is important as they usually play important role in the assessment, planning and management of abnormal placentation cases and development and implication of massive blood transfusion protocol with frequent assessment of blood loss $[14,15]$.

\section{Conclusion}

This report highlights the importance of prenatal diagnosis of placenta percreta and the preparation and planning to manage these cases, as well as the importance of multidisciplinary care team approach (high risk obstetrician, anesthesiologist, urologist, general surgeon, interventional radiologist and neonatologist) and the communication between the team members. The keys for successful management include:

- Assessment of risk factors and Prenatal Diagnosis, which permits evaluation, optimisation and planning to improve the outcome and decrease the complications.

- Multidisciplinary Care Team Approach, with involvement of the most expert specialists in the management of these cases, and the teamwork with precise communications between the team members is of great value. This might contribute to decrease the rate of morbidity.

- Location of surgery is an important part of planning, since logistics, access to facilities and proximity to the ICU can play an important role in successful management of these cases.

- Early involvement of expert anesthesiologist to be part of proactive team is important as they usually play important role in the assessment, planning and management of abnormal placentation cases.

- Development and implication of massive blood transfusion protocol with frequent assessment of blood loss, including frequent monitoring of relevant laboratory values which helps in the management of massive blood transfusion.

\section{References}

1. Flood KM, Said S, Geary M, Robson M, Fitzpatrick C, et al. (2009) Changing trends in peripartum hysterectomy over the last 4 decades. Am J Obstet Gynecol 200: 632.

2. Wu S, Kocherginsky M, Hibbard JU (2005) Abnormal placentation: twenty-year analysis. Am J Obstet Gynecol 192: 1458-1461.

3. Awan N, Bennett MJ, Walters WA (2011) Emergency peripartum hysterectomy: a 10-year review at the Royal Hospital for Women, Sydney. Aust N Z J Obstet Gynaecol 51: 210-215.

4. Kwee A, Bots ML, Visser GH, Bruinse HW (2006) Emergency peripartum hysterectomy: A prospective study in The Netherlands. Eur J Obstet Gynecol Reprod Biol 124: 187-192.

5. Rao KP, Belogolovkin V, Yankowitz J, Spinnato JA 2nd (2012) Abnormal placentation: evidence-based diagnosis and management of placenta previa, placenta accreta, and vasa previa. Obstet Gynecol Surv 67: 503-519.

6. Publications Committee, Society for Maternal-Fetal Medicine, Belfort MA (2010) Placenta accreta. Am J Obstet Gynecol 203: 430-439.

7. Kuczkowski KM (2011) A review of current anesthetic concerns and concepts for cesarean hysterectomy. Curr Opin Obstet Gynecol 23: 401-407.

8. Kocaoglu N, Gunusen I, Karaman S, Ergenoglu AM, Firat V (2012) Management of anesthesia for cesarean section in parturients with placenta previa with/without placenta accreta: a retrospective study. Ginekol Pol 83: 99-103.

9. Ahonen J, Jokela R (2005) Recombinant factor VIla for life-threatening post-partum haemorrhage. Br J Anaesth 94: 592-595.

10. Vincent JL, Rossaint R, Riou B, Ozier Y, Zideman D, et al. (2006) Recommendations on the use of recombinant activated factor VII as an adjunctive treatment for massive bleeding--a European perspective. Crit Care 10: R120.

11. Gungorduk K, Yildirim G, Asicioglu O, Gungorduk OC, Sudolmus S, et al. (2011) Efficacy of intravenous tranexamic acid in reducing blood loss after elective cesarean section: a prospective, randomized, double-blind, placebo-controlled study. Am J Perinatol 28: 233-240.

12. Novikova N, Hofmeyr GJ (2010) Tranexamic acid for preventing postpartum haemorrhage. Cochrane Database Syst Rev CD007872.

13. Clausen C, Lönn L, Langhoff-Roos J (2014) Management of placenta percreta: a review of published cases. Acta Obstet Gynecol Scand 93: 138-143.

14. Warshak CR, Ramos GA, Eskander R, Benirschke K, Saenz CC, et al. (2010) Effect of predelivery diagnosis in 99 consecutive cases of placenta accreta. Obstet Gynecol 115: 65-69.

15. Eller AG, Bennett MA, Sharshiner M, Masheter C, Soisson AP, et al. (2011) Maternal Morbidity in Cases of Placenta Accreta Managed by a Multidisciplinary Care Team Compared With Standard Obstetric Care. Obstet Gynecol 117: 331-337. 